\title{
Evolving in silico Bistable and Oscillatory Dynamics for Gene Regulatory Network Motifs
}

\author{
Yaochu Jin, Senior Member, IEEE, Bernhard Sendhoff, Senior Member, IEEE
}

\begin{abstract}
Autoregulation, toggle switch and relaxation oscillators are important regulatory motifs found in biological gene regulatory networks and interesting results have been reported on theoretical analyses of these regulatory units. However, it is so far unclear how evolution has shaped these motifs based on elementary biochemical reactions. This paper presents a method of designing important dynamics such as bistability and oscillation with these network motifs using an artificial evolutionary algorithm. The evolved dynamics of the network motifs are then verified when the initial states and the parameters of the network motifs are perturbed. It has been found that while it is straightforward to evolve the switching behavior, it is difficult to evolve stable oscillatory dynamics. We show that a higher Hill coefficient will facilitate the generation of undamped oscillation, however, an evolutionary path that can lead to a high Hill coefficient remains an open question for future research.
\end{abstract}

\section{INTRODUCTION}

Gene regulatory networks play a central role in the evolution and development of biological systems. In order to understand natural evolution, it is of essence to understand the structure and dynamics of gene regulatory networks. There are two possible ways to achieve this. On the one hand, the availability of huge amount of microarray data for gene expression makes it possible to reconstruct the underlying gene regulatory network for a particular function using mathematical methods [1], [2]. Two main difficulties arise in building mathematical models of gene regulatory networks. First, the microarray data are often very noisy. Second, the dimension of the model, i.e., the number of genes involved, is extremely high, whereas the number of records are few.

On the other hand, a small number of sub-networks, also known as network motifs, occur very often in complex gene regulatory networks. These network motifs serve as building blocks of regulatory networks and the dynamics of the whole networks can be analyzed by looking at these motifs. Detection and analysis of the regulatory motifs in biological systems has now become one important research topic in systems biology [3], [4].

While most work concentrates on the analysis of dynamics of network motifs, this paper investigates in silico whether it is able to evolve the desired dynamics given the structure of a network motif. To this end, we employ an evolution strategy, one of the widely used artificial evolutionary algorithms [5], to evolve the parameters of the given network motifs. Though evolution of the desired dynamics for a given network motif

Yaochu Jin and Bernhard Sendhoff are with the Honda Research Institute Europe, 63073 Offenbach, Germany. Email: yaochu.jin@honda-ri.de. appears straightforward at the first sight, we find out that it is nontrivial to evolve undamped oscillations, i.e., limit cycles. Our experimental results reveal that oscillation seems to be relatively more easily evolvable for large Hill coefficients. In addition, only a limited range of oscillation periods can be evolved. Finally, once undamped oscillation is evolved, it is quite robust against perturbations in the parameters of the network motifs, but may be sensitive to initial states.

Evolving dynamics for gene regulatory networks in silico has been reported in the literature. In [6], both bistable switches and oscillators are evolved based on a number of predefined basic biochemical reactions. In that work, the fitness function is the difference between the real output and the desired amplitude $A_{1}$ at half-integer periods, and the difference between the real output and the desired amplitude $A_{2}$ at integer periods. However, it was indicated in [7] that the results reported in [6] are not easily reproducible, which implies that successful evolution of sustained oscillation is sensible to experimental setups. In [7], a correlation based fitness function has been suggested, though no definite conclusion can be drawn on its influence on the successful evolution of oscillators. Similar work has also been reported in [8], where two different fitness functions are suggested for evolving oscillation. The first approach is the same as in [6], and the second one is based on the condition for generating Hopf bifurcation. In [9], oscillatory dynamics is evolved using the Biosys model developed in [10].

This paper is structured as follows. In Section II, a brief introduction to gene expression and its mathematical modeling is provided along with a description of the mathematical models of the network motifs we study in the paper. An evolution strategy is presented in Section III. Experimental results are presented and discussed in Section IV, and Section $\mathrm{V}$ concludes this paper.

\section{Regulatory Network Motifs}

A simplified view of gene expression consists of two main steps, namely, transcription and translation, where in transcription an mRNA copy of the DNA is made, and in translation, proteins are synthesized from mRNA. Whether a structural gene will be expressed (activated) or not (repressed) is decided by a series of interactions within the regulatory network.

If the expression of a gene can be regulated (activated or repressed) by the protein produced by itself, it is termed autoregulation. The mathematical model of gene expression 


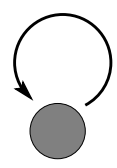

(a)

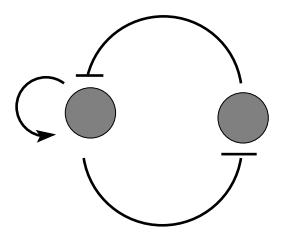

(b)

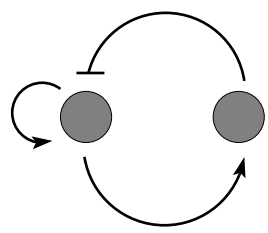

(c)
Fig. 1. Three regulatory motifs studied in the paper. (a) PAR, (b) Toggle switch with PAR, and (c) relaxation oscillation.

with autoregulation can be described by:

$$
\begin{aligned}
\frac{d[R]}{d t} & =-\gamma_{R}[R]+\alpha_{R} H([P]), \\
\frac{d[P]}{d t} & =-\gamma_{P}[P]+\alpha_{P}[R],
\end{aligned}
$$

where, $[R]$ and $[P]$ are the concentration of mRNA and protein, respectively, $\gamma_{R}$ and $\gamma_{P}$ are the decay rate of the mRNA and protein, $\alpha_{R}$ and $\alpha_{P}$ are the synthesis rate of the mRNA and protein, $H(X)$ is the Hill function. If the autoregulation is a repression, also know as negative autoregulation, the Hill function can be described by

$$
H_{r}(x)=\frac{\beta}{\theta^{n}+x^{n}},
$$

and if the autoregulation is activation, the Hill function can be written as:

$$
H_{a}(x)=\frac{\beta x^{n}}{\theta^{n}+x^{n}},
$$

where $\beta$ is the activation coefficient, $\theta$ is the threshold, $n$ is the Hill coefficient.

Three network motifs will be studied in the paper, including the positive autoregulation (PAR), toggle switch with positive autoregulation, and relaxation oscillation, as shown in Fig. 1 (a), (b), and (c), respectively. For the sake of convenience, we provide again the mathematical model of the three motifs as follows. The mathematical models of a PAR can be given by:

$$
\begin{aligned}
& \dot{x}_{1}=-a_{11} x_{1}+a_{12} H_{a}\left(x_{2}\right), \\
& \dot{x}_{2}=a_{21} x_{1}-a_{22} x_{2},
\end{aligned}
$$

where $x_{1}$ corresponds to $[R]$, is the concentration of mRNA, and $x_{2}$ corresponds to $[P]$, the concentration of protein. There are different ways to combine multiple regulatory inputs to the promoter of a particular gene, which lead to slightly different mathematical models. In [3], logic functions such as logic AND or OR are suggested to combine multiple regulatory inputs. In this work, we suppose the regulatory inputs are additive. In this case, the dynamics of the toggle switch with PAR can be described by:

$$
\begin{aligned}
& \dot{x}_{1}=-a_{11} x_{1}+a_{12} H_{a}\left(x_{2}\right), \\
& \dot{x}_{2}=a_{21} x_{1}-a_{22} x_{2}+a_{23} H_{r}\left(x_{3}\right), \\
& \dot{x}_{3}=a_{32} H_{r}\left(x_{2}\right)-a_{33} x_{3},
\end{aligned}
$$

where $x_{1}$ represents the mRNA concentration, and $x_{2}, x_{3}$ the concentration of two proteins.

And finally, the oscillation motif can be expressed by:

$$
\begin{aligned}
& \dot{x}_{1}=-a_{11} x_{1}+a_{12} H_{a}\left(x_{2}\right), \\
& \dot{x}_{2}=a_{21} x_{1}-a_{22} x_{2}+a_{23} H_{r}\left(x_{3}\right), \\
& \dot{x}_{3}=a_{32} H_{a}\left(x_{2}\right)-a_{33} x_{3} .
\end{aligned}
$$

Similar to the toggle switch, $x_{1}$ represents the mRNA concentration, and $x_{2}, x_{3}$ denote the concentration of two proteins.

\section{Evolution StRATEGY}

Evolution strategies are one of the main streams of artificial evolutionary algorithms that are very effective for evolving real-valued parameters. Since the structure of the regulatory motifs is fixed, and only the parameters are evolved in this work, we adopt a canonical evolution strategy for evolving the desired dynamics. In a canonical evolution strategy (ES), the mutation of the objective parameters is performed by adding an $N\left(0, \sigma_{i}^{2}\right)$ distributed random number. The stepsizes $\sigma_{i}$ are also encoded in the genotype and subject to mutations. The ES used in this work can be described as follows:

$$
\begin{aligned}
\mathbf{x}(t) & =\mathbf{x}(t-1)+\tilde{\mathbf{z}} \\
\sigma_{i}(t) & =\sigma_{i}(t-1) \exp \left(\tau^{\prime} z\right) \exp \left(\tau z_{i}\right) ; i=1, \ldots, n,
\end{aligned}
$$

where $\mathbf{x}$ is an $n$-dimensional parameter vector to be evolved, $\tilde{\mathbf{z}}$ is an $n$-dimensional random number vector with $\tilde{\mathbf{z}} \sim$ $N\left(\mathbf{0}, \boldsymbol{\sigma}(t)^{2}\right), z$ and $z_{i}$ are normally distributed random numbers with $z, z_{i} \sim N(0,1)$. Parameters $\tau, \tau^{\prime}$ and $\sigma_{i}$ are called strategy parameters, where $\sigma_{i}$ is mutated as in equation (14) and $\tau, \tau^{\prime}$ are constants as follows:

$$
\tau=(\sqrt{2 \sqrt{n}})^{-1} ; \tau^{\prime}=(\sqrt{2 n})^{-1}
$$

Two selection schemes have been proposed in evolution strategies, known as comma and plus strategies. Suppose there are $\mu$ and $\lambda$ individuals in the parent and offspring population, usually $\mu \leq \lambda$. In the comma strategy, $\mu$ parent individuals are selected only from the $\lambda$ offspring individuals, which is usually noted as $(\mu, \lambda)$-ES. In the plus strategy, $\mu$ parent individuals are selected from a combination of $\mu$ parent individuals and $\lambda$ offspring individuals, which is noted as $(\mu+\lambda)$-ES. In our study, the $(\mu, \lambda)$-ES is adopted.

In the evolution, all parameters in the regulatory models, i.e., four decay and synthesis rates in PAR model and seven in the toggle and oscillation models, together with the activation coefficient $(\beta)$, the threshold $(\theta)$, and Hill coefficient $(n)$ are encoded in the genome and subject to evolution.

\section{Simulation Results}

\section{A. Experimental setup}

A $(50,300)$-ES has been adopted in our experiments. All parameters to be evolved are randomly initialized between 0 and 4. According to the physical meaning of the parameters, a lower bound is set to 0 for all parameters, but no upper 
bound is given. The initial stepsize is set to 0.1. In all simulations, 1000 generations are run for each case.

To evolve the bistable dynamics for the PAR, the initial state of the regulatory model is randomly set between 0 and 4. The target function of the protein concentration of $x_{2}$ for the PAR is as follows:

$$
x_{2}^{d}(\infty)= \begin{cases}0, & \text { if } x_{2}(0)<2 \\ 4, & \text { if } x_{2}(0) \geq 2\end{cases}
$$

For the toggle switch motif, the same initial states are set and the target of $x_{3}$ is defined similar to equation (16).

The target function for $x_{3}$ in evolving oscillation is defined by a sinus function as follows:

$$
x_{3}^{d}(t)=\sin (2 \pi t / \mathrm{T})+1.0
$$

where $t$ is time instant, and $T$ is the period of the desired oscillation.

The evolution of oscillation is more tricky. As indicated in [7], the success rate of achieving the desired dynamics is very low when both the structure and parameters are evolved. we find even in our case, where the structure of the network motif is predefined, is it not straightforward to evolve limit cycles. This issue will be discussed further in Section II.C.

\section{B. Evolving Bistability}

The fitness profile including the mean fitness (dotted line) and best fitness (solid line) in evolving bistability for the PAR is presented in Fig. 2. After evolution, the dynamics of the system is verified using 50 random initial states. The state-space trajectories of $x_{1}$ and $x_{2}$ are given in Fig. 3(a), and the time course of state $x_{2}$ is provided in Fig. 3(b). It can be seen that the evolved bistability is very robust to different initial states. Besides, we also found that the evolution of bistability has been successful for all test runs.

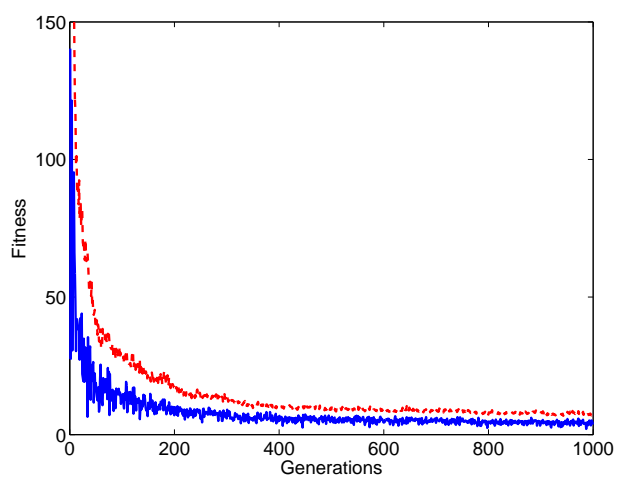

Fig. 2. Mean and best fitness in evolving switching behavior for the positive autoregulation motif.

Similar results have been obtained for the toggle switch with positive autoregulation. Its state-space trajectories and time course are presented in Figs. 4.

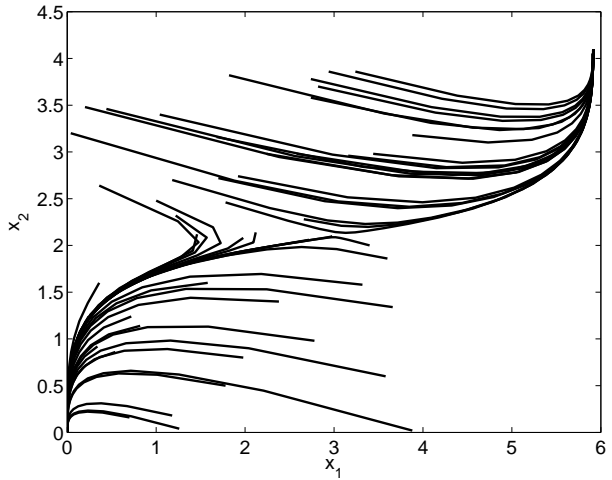

(a)

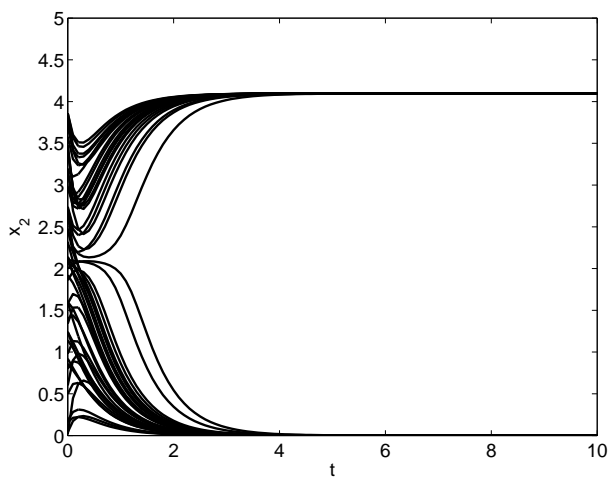

(b)

Fig. 3. Dynamics of the evolved positive autoregulation motif for different initial states. (a) state-space trajectories, and (b) time course.

\section{Evolving Oscillation}

In the first runs, we were not able to achieve a limit cycle in 10 independent runs. We then check the success rate for six different desired oscillation periods $T$ and four different encoding schemes for the Hill coefficient $n$ :

$$
n=N \times g_{n},
$$

where $g_{n}$ is the gene that encodes the Hill coefficient in the chromosome, $N \geq 1$ is a constant that adjusts the coding range of this gene. Clearly, if $N=\infty$, the Hill function will always be a step function, no matter how $g_{n}$ changes. In our experiments, simulations are conducted for $N=1,5,10, \infty$. The success rates for different cases are show in Fig. 5, where 0 means no success in ten independent runs, and 1 means all ten runs have succeeded in achieving stable oscillation. Note that in many of unsuccessful runs, the state $\left(x_{3}\right)$ does oscillate, but becomes asymptotically stable, see e.g., in Fig. 6. In this case, we do not count it as a successful run.

From Fig. 5, we find that the larger the Hill coefficient, the easier it is to evolve undamped oscillation. When the Hill function becomes a step function $(n=\infty)$, the success rate becomes one for desired oscillation periods ranging from 3 to 9. Meanwhile, we find that although there is no upper bound for the Hill coefficient $n$ during the evolution, it is not straightforward for the evolutionary algorithm to find an 


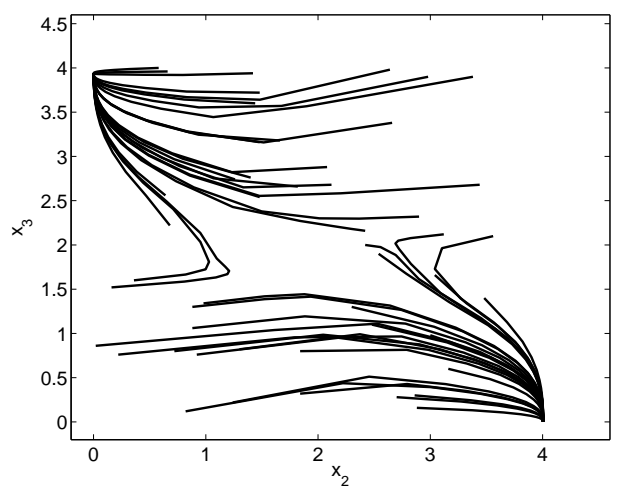

(a)

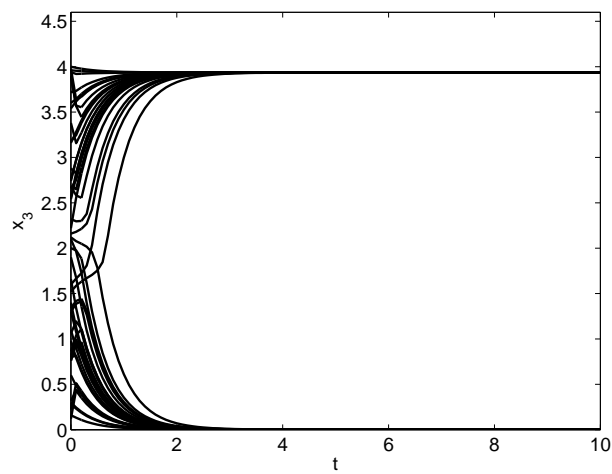

(b)

Fig. 4. Dynamics of the evolved toggle switch with PAR for different initial states. (a) state-space trajectories, and (b) time course.

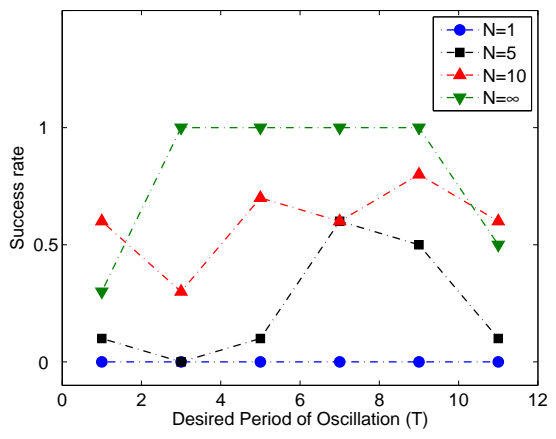

Fig. 5. Success rate in evolving stable oscillation for different $T$ and $N$.

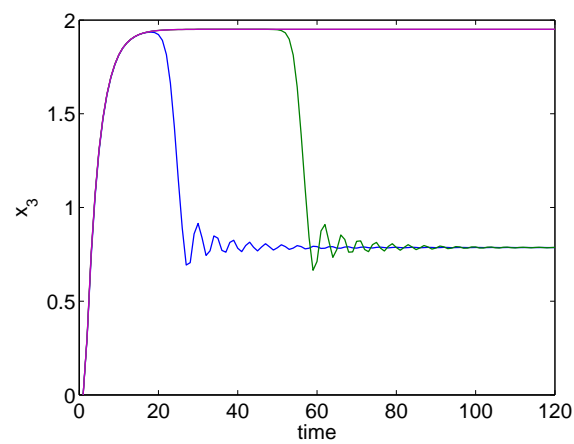

Fig. 6. Evolved damped oscillation when $N=5$ and $T=7$. evolutionary path toward a larger $n$ that facilitates a sustained oscillation. This problem may be attributed to the fitness function used: unlike in evolving the switching behavior, a smaller (better) fitness value does necessarily lead to a stable oscillation.

For the successfully evolved oscillators, We first verify the robustness of the oscillatory dynamics against different initial states after evolution. Five different initial states are tested for two evolved oscillators generated in two independent runs when $N=10$ for desired oscillation period of $T=5$. Although the evolved dynamics appears very similar, their behaviors for $x_{1}(0)=1.0,2.0,3.0,4.0,5.0$ are different, refer to Fig. 7. In Fig. 7(a), all five initial states of $x_{1}$ lead to stable oscillation, while in Fig. 7(b), the evolved motif can produce stable oscillation only for two of the five initial states of $x_{1}$.

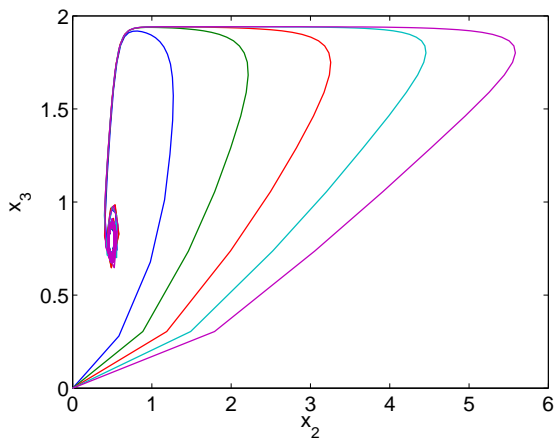

(a)

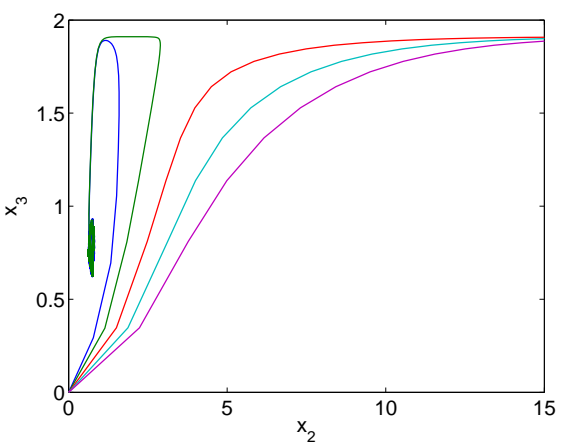

(b)

Fig. 7. Two oscillators evolved in two independent runs for $T=5$, $N=10$. In (a), the evolved oscillator shows undamped oscillation for all the five initial states, whereas in (b), the oscillator exhibits limit cycle for only two of the initial states.

We then check how well the evolved oscillator is able to approximate the desired oscillation period. The time course of $x_{3}$ of two evolved oscillators for a desired oscillation period $T=5, N=10$ and $T=9, N=\infty$ are presented in Fig. 8 and Fig. 9, respectively. We can see from the figures that the evolved oscillations is roughly the same as the desired one. The state-space trajectory of these two oscillators are presented in Fig. 10 and Fig. 11, respectively.

We finally compare the robustness of the evolved oscillators against perturbations in the parameters for different Hill 


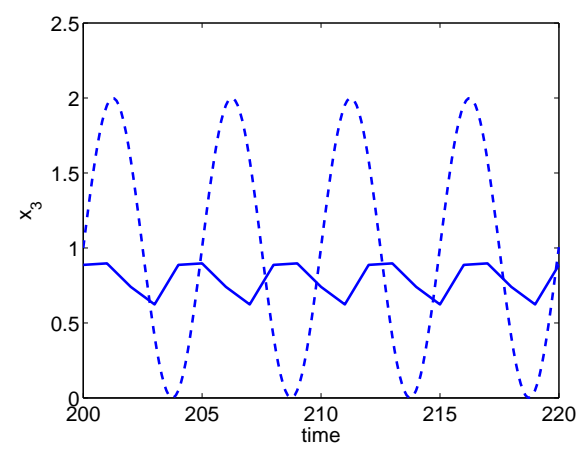

Fig. 8. Real and the desired states of $x_{3}$ of an evolved oscillator. $T=5$, $N=10$.

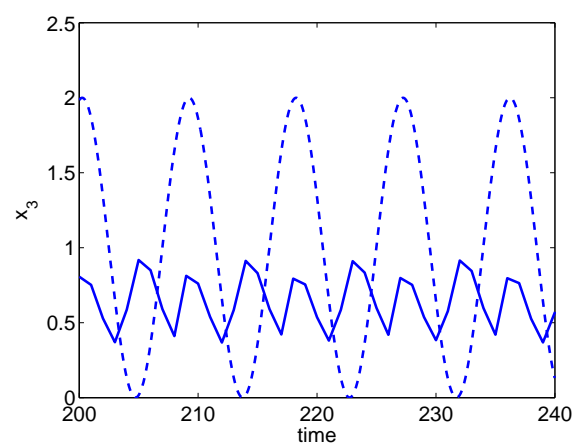

Fig. 9. Real and the desired states of $x_{3}$ of an evolved oscillator. $T=9$, $N=\infty$.

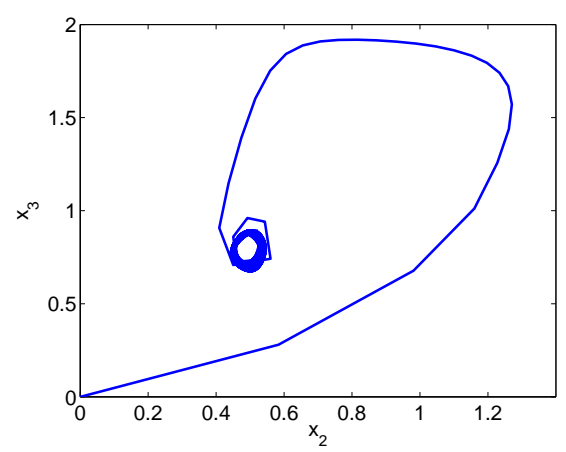

Fig. 10. Oscillation dynamics of the evolved oscillator for $N=10, T=5$.

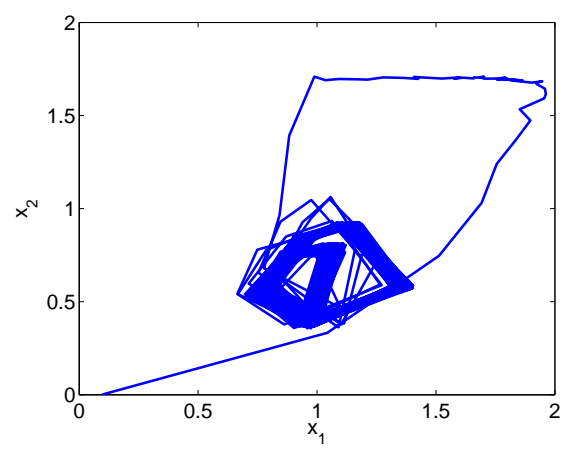

Fig. 11. Oscillation dynamics of the evolved oscillator for $N=\infty, T=9$.

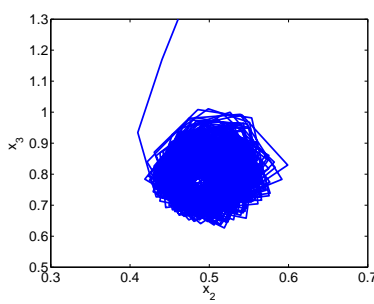

(a)

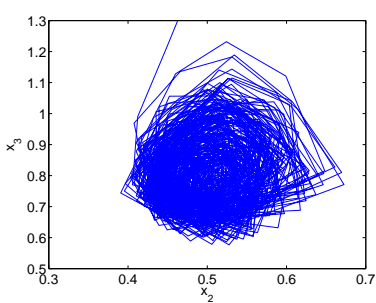

(c)

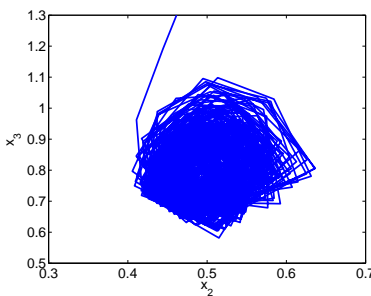

(b)

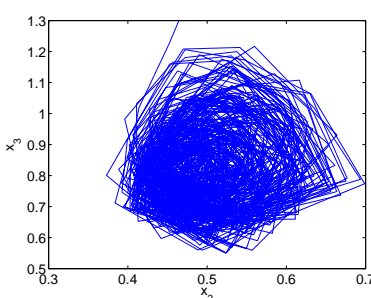

(d)
Fig. 12. The oscillation dynamics when the parameters of the evolved oscillator $(n=10.7)$ are perturbed with various noise strength. (a) $5 \%$, (b) $10 \%$, (c) $15 \%$, and (d) $20 \%$

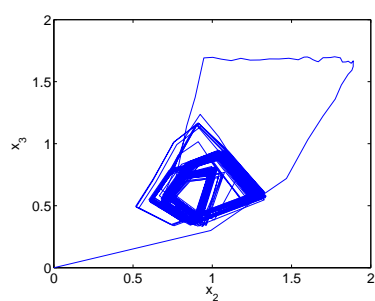

(a)

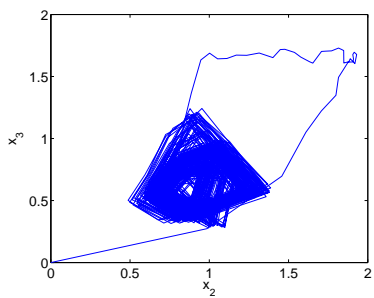

(c)

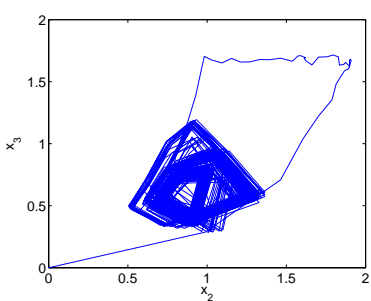

(b)

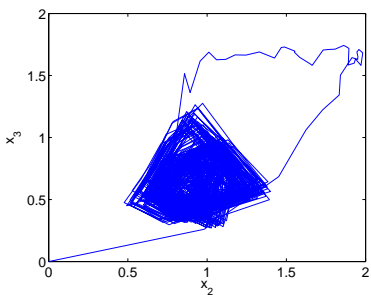

(d)
Fig. 13. The oscillation dynamics when the parameters of the evolved oscillator $(n=\infty)$ are perturbed with various noise strength. (a) $5 \%$, (b) $10 \%$, (c) $15 \%$, and (d) $20 \%$.

coefficients. After evolution, noise with a strength varying from $5 \%$ to $20 \%$ is added to the parameters of the oscillators, one with a Hill coefficient $n=10.7$, and the other with $n=\infty$. The state-space dynamics of the two oscillators in the presence of noise in parameters are presented in Fig. 14 when $n=10.7$, and Fig. 13, whereas the time courses of $x_{3}$ are given in Fig. 14 and Fig 15, respectively. From these figures, we can conclude that the evolved oscillators are quite robust to perturbations in the parameters. However, it appears that the oscillator with an infinite Hill coefficient shows more robust oscillatory behavior than the one with a smaller Hill coefficient. 


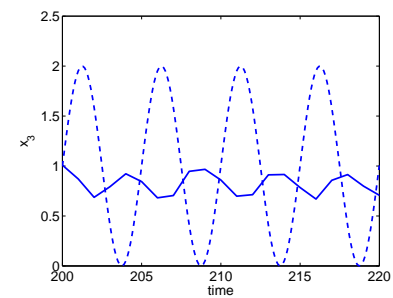

(a)

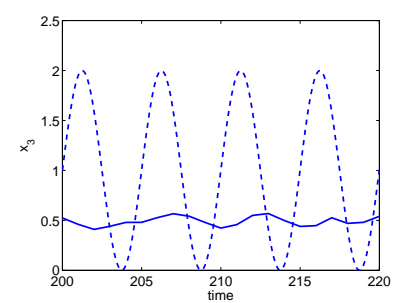

(c)

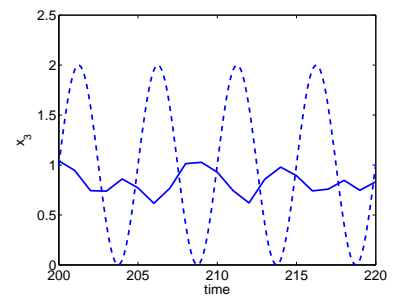

(b)

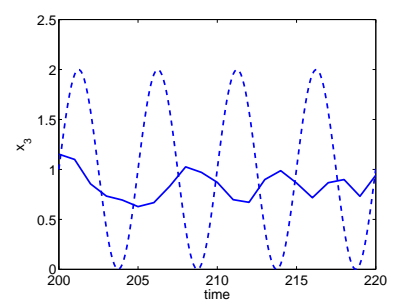

(d)
Fig. 14. Time course of $x_{3}$ of the oscillator when the parameters of the evolved oscillator $(n=10.7)$ are perturbed with various noise strength. (a) $5 \%$, (b) $10 \%$, (c) $15 \%$, and (d) $20 \%$.

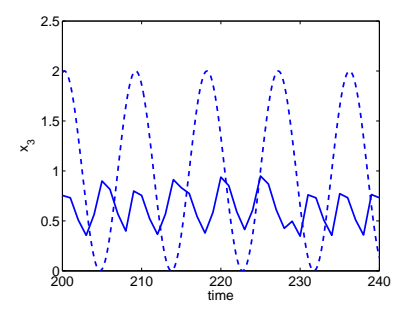

(a)

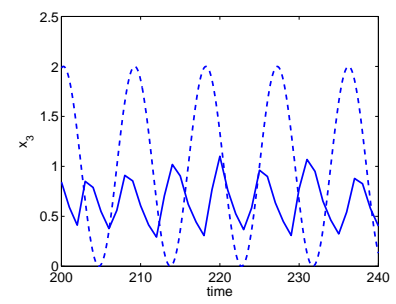

(c)

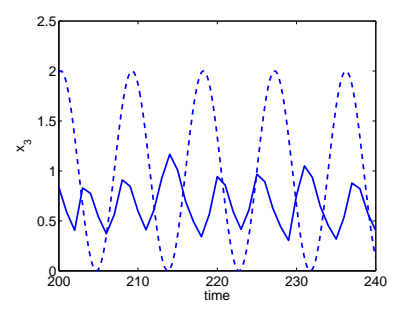

(b)

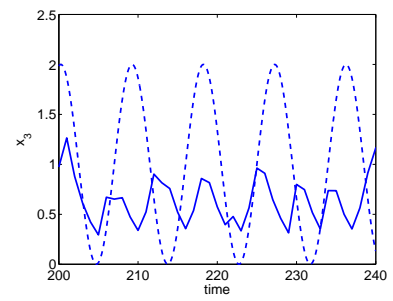

(d)
Fig. 15. Time course of $x_{3}$ of the oscillator when the parameters of the evolved oscillator $(n=\infty)$ are perturbed with various noise strength. (a) $5 \%$, (b) $10 \%$, (c) $15 \%$, and (d) $20 \%$.

\section{CONCLUSions}

This paper presents a method for evolving in silico bistable and oscillatory dynamics for three regulatory networks motifs, i.e., the positive autoregulation (PAR), the toggle switch with PAR, and the relaxation oscillation. We find that while it is straightforward to evolve bistability, it is nontrivial to evolve sustainable oscillation. We find that in general, it is easier to achieve stable oscillation for large Hill coefficients (e.g., when the Hill function becomes the step function). However, it is not straightforward for the evolutionary algorithm to evolve a large Hill coefficient, even no upper bound is set for the parameters. This may be attributed to the simple fitness function used in this work. Alternatively, we can use other fitness functions such as correlation studied in [7], or a more loosely constrained fitness function suggested in [11]. However, as found in above works, it is nevertheless nontrivial to evolve stable oscillatory dynamics. We also show that the evolved oscillators are quite robust to perturbations in parameters, though they can be sensitive to initial states.

One main constraint of this paper is that the structure of the motifs is predefined and only their parameters are evolved. On the other hand, it has been argued both in [11] and [7] that evolving oscillators from scratch is a daunting task. Therefore, further efforts are needed to understand the evolution of most basic regulatory motifs. On the basis of these understandings, we can then study how to evolve regulatory complex dynamics given that some most basic motifs, such as the autoregulation, are available.

\section{REFERENCES}

[1] H. de Jong, "Modeling and simulation of genetic regulatory systems: A literature review," Journal of Computational Biology, vol. 9, no. 1, pp. 67-103, 2002.

[2] T. Lee, D. Tu, C. Tan, and L. You, "Modeling cellular networks," in Bioinformatics: Foundations and Applications, 2007, ch. 6.

[3] U. Alon, An Introduction to Systems Biology: Design Principles of Biological Circuits. Boca Raton: Chapman \& Hall, 2007.

[4] _ - "Network motifs: theory and experimental approaches," Nature Review Genetics, vol. 8, pp. 450-461, 2007.

[5] T. Bäck, Evolutionary Algorithms in Theory and Practice: Evolution Strategies, Evolutionary Programming, Genetic Algorithms. Oxford University Press, 1996.

[6] P. Francois and V. Hakim, "Design of genetic networks with specified functions by evolution in silico," Proceedings of the National Academy of Sciences of USA, vol. 101, no. 2, pp. 580-585, 2007.

[7] D. Chu, "Evolving genetic regulatory networks for systems biology," in Congress on Evolutionary Computation. IEEE Press, 2007, pp. $875-882$.

[8] S. Paladugu, V. Chickarmane, A. Dekard, J. Frumkin, M. MaCormarck, and H. Sauro, "In silico evolution of functional modules in biochemical networks," IEEE Proceedings on Systems Biology, vol. 153, no. 4, pp. 223-235, 2006.

[9] J. Knabe, C. Nehaniv, M. Schilstra, and T. Quick, "Evolving biological clocks using genetic regulatory networks," in Artificial Life X. MIT Press, 2006, pp. 15-21.

[10] T. Quick, C. Nehaniv, K. Dautenhahn, and G. Roberts, "Evolving embodied genetic regulatory network-driven control systems," in European Conference on Artificial Life (ECAL'03), ser. Lecture Notes in Artificial Intelligence 2801. Springer, 2003, pp. 266-277.

[11] B. Drennan and R. Beer, "Evolution of repressilators using a biologically-motivated model of gene expression," in Artificial Life X. MIT Press, 2006, pp. 22-27. 